\title{
Household Water Treatment and Storage Practices Among Residents in Mogadishu Somalia
}

\author{
Fatima Hassan Adam ${ }^{1, ~ *}$, Abdirizak Mohamud Yusuf ${ }^{2, *}$, Mohamed Ahmed Alasow ${ }^{3}$, Said Hussein Gedi ${ }^{4}$ \\ ${ }^{1}$ Postgraduate and Research Studies Centre, Benadir University, Mogadishu, Somalia \\ ${ }^{2}$ Department of Public Health, Ministry of Health, Mogadishu, Somalia \\ ${ }^{3}$ Faculty of Health Science, Benadir University, Mogadishu, Somalia \\ ${ }^{4}$ Department of Ophthalmology, Faculty of Medicine, Somali National University, Mogadishu, Somalia
}

Email address:

abdirizak992@gmail.com (A. M. Yusuf)

${ }^{*}$ Corresponding author

\section{To cite this article:}

Fatima Hassan Adam, Abdirizak Mohamud Yusuf, Mohamed Ahmed Alasow, Said Hussein Gedi. Household Water Treatment and Storage Practices Among Residents in Mogadishu Somalia. Science Journal of Public Health. Vol. 9, No. 5, 2021, pp. 173-179. doi: 10.11648/j.sjph.20210905.16

Received: September 22, 2021; Accepted: October 14, 2021; Published: October 28, 2021

\begin{abstract}
Background: Consumption of contaminated water can affect human health and even lead to high morbidity and mortality, particularly among under-five children. Thus, determining household water treatment and proper management is vital to eliminate contamination. This study aimed to assess the household water treatment and storage practices among Warta-Nabada residents in Mogadishu, Somalia. Methods: A community-based cross-sectional study was conducted in 386 households from the April to June 2018 period. A multistage cluster sampling technique was used to select sample units from the households to obtain accurate data. Data were collected using structured questionnaires. Also, SPSS version 21 was used for data entry and analysis, respectively. The univariate, bivariate, and multivariate levels were analyzed to see a significant association between variables. The statistical significance was declared at a value $<0.05$ with $95 \%$ confidence intervals in the final model. Results: The majority of participants, 291 (75.4\%), used plastic containers for water storage at a household level, while 48 (12.4\%) used clay pots, followed by $47(12.2 \%)$ cement tanks. Most containers used for water storage were plastic containers due to their availability and low cost. Participants who had containers were 227 (58.8\%). Approximately 114 (29.5\%) had two containers. The number of people who practice boiling water was relatively low $(15.8 \%)$. The distance of the water source from household $143(37.0 \%)$ were more than 50 meters, regards to methods for water treatment, $91(23.6 \%)$ were used for chlorination, and $61(15.8 \%)$ were used for boiling, but $45(11.7 \%)$ were used for filtration, and $22(5.7 \%)$ used solar disinfection as well. Therefore, the most common water treatment method used was chlorination, accounting for $(23.6 \%)$ of the total water treated. Conclusion: According to the results, it can be concluded there were significant variables for age, marital status, occupational, and education of the husband of the household water treatment level. When there was a high risk of acute water diarrhea or cholera, drinking water chlorination, boiling, and filtration were the most common household water treatment practices. Among a group of good practices, there were (73\%) while the poor practices group had (27\%). However, the majority of residents practice precisely. This study recommended developing a comprehensive national plan for scaling up the household water treatment system with a holistic approach, allowing adequate service provision and addressing the need to provide for the most vulnerable groups with better practices.
\end{abstract}

Keywords: Household Water Treatment, Residents, Knowledge Level, Practices, Water Storage

\section{Introduction}

Today, millions of people worldwide do not have access to safe water, particularly among the population of developing and underdeveloped countries [1]. A study conducted by
WHO and UNICEF highlighted that more than 700 million people in the world do not access improved drinking water sources, due to the nature of their construction, are protected from outside contamination. Drinking water quality has shown that hundreds of millions of people with "improved" 
drinking water do not have access to a source that is microbiologically safe to drink [2]. Globally, household water treatment is not feasible at the household level, indicating that they are left with the responsibility of needing to collect, treat, and store their water for safety and improved health [3]. Although many countries' groundwater is inaccessible or contaminated, these users depend on household water treatment systems for safe drinking water storage because 663 million people lack improved drinking water sources [4]. Therefore, contaminated water resulting from unsafe consumer storage and poor handling practices at the household level causes diarrhea [5].

Approximately half of the people using unimproved drinking water sources live in Sub-Saharan Africa, while one-fifth live in Southern Asia. Furthermore, Sub-Saharan Africa has made little progress, with $43 \%$ of its population having access to improved drinking water, while some developing countries in other regions are said to be falling behind the MDGs standards for drinking water that needs further consideration [6]. These household water treatment systems aim to provide safe drinking water affordably and sustainably [7]. However, the inhabitants can manage their use of water and easy to avoid water-borne diseases [8].

The regular practice of household-level water treatment is crucial in reducing the occurrence of diarrheal and other devastating illnesses [9]. Currently, 842000 people and 36 1000 children aged less than five years are estimated to die from diarrhea due to unsafe drinking water, poor sanitation, and hand hygiene practices. Although communities may have access to piped water at home, it may be contaminated by defects in the distribution system or improper storage [10]. A recent study in Somalia highlighted that $(66.1 \%)$ are not treating their water instead of $(31.5 \%)$ practicing any water treatment method. Water chlorination was used as a disinfectant by more than $(27.3 \%)$. Around $(4.0 \%)$ used boiling as a means of water treatment as well. Among those, $(38.8 \%)$ believed that water is safe naturally. Most of the households $(97.6 \%)$ had drinking water containers. Approximately $(80.6 \%)$ of families indicated that they use Jerri cans for water collection and storage, and only (3.6\%) have a bucket without a lid for water storage [11]. There is a commitment to safe household water treatment and storage, which has led to the formation of the WHO-sponsored international network promotion that has brought together several stakeholders to improve household water management as a component of water, sanitation, and hygiene programs [12]. This study aimed to assess household water and storage practices in Warta-Nabada district, Mogadishu, Somalia.

\section{Methods and Materials}

\subsection{Study Design, and Site}

Community-based cross-sectional study was conducted during the period between April to June 2018 in WartaNabada, Mogadishu, Somalia.

\subsection{Sample Size Calculation}

The sample size was calculated using Slovene's formula, which is $\mathrm{n}=\mathrm{N} / 1+\left(\mathrm{N}^{*} \mathrm{e}^{2}\right)$, where $\mathrm{n}=$ sample size and $\mathrm{N}=$ population and $\mathrm{e}=5 \%$ margin of error $\mathrm{N}=10,600 / 1+1 / 10,600 * 0.0025)=386$ households.

\subsection{Sampling Technique}

A multistage cluster sampling technique was used to select sample units from the sampled households: In the first stage, the sampling frame consists of five sections. In the second stage of each selected section, respondents were selected based on sub-sections. The third stage, from each selected sub-section, was divided into blocks. From each block, the study participants have been selected randomly through systematic sampling.

\subsection{Data Collection Techniques and Instruments}

This quantitative study used a structured questionnaire for interviews. Both closed-ended and open-ended questions were utilized for data collection. During the data, collection interviews were conducted in the local language and then professionally translated to English. Three qualified experts validated the translation process to ensure validity, and they prepared the manuscript in English.

\subsection{Data Analysis}

The Statistical Package for Social Sciences (SPSS) software, 21 versions, was used to code, enter, and analyze data. The univariate analysis was used to provide frequency, percentages and draw various charts. The logistic regression analysis and odds ratios were also used to determine the relationships between variables. The level of significance was set at $\mathrm{P}<0.05$ and $95 \%$ confidence intervals.

\subsection{Ethical Considerations}

Ethical approval for the study was obtained from the Benadir University, Postgraduate Studies and Research Centre Ethics Committee. Every participant gave their informed consent before participating in the study. Participants' information and identifiers were appropriately reserved.

\section{Results}

\subsection{Socio-demographic Characteristics}

Table 1 shows the age distribution of the respondents was $258(66.8 \%)$ and ranged from 16-39 years, among 128 $(33.2 \%)$ were $40-86$ years, respectively. The majority were aged between 16 and 39 years, which is about $258(66.8 \%)$, mainly related to those interested in a household water treatment. These findings also revealed that 166 (43.3\%) participants were males, while $220(57.0 \%)$ were female. Over, $221(57.3 \%)$ of the respondents were married, followed by $98(25.4 \%)$ of the single participants, and $36(9.3 \%)$ were divorced. Only 31 (8.0\%) were widowed. Among 151, 
(39.1\%) could not read, but $86(22.3 \%)$ could read and write. Only $69(17.9 \%)$ were primary, $43(11.1 \%)$ were secondary, and $37(9.6 \%)$ were university level. Therefore, $121(31.3 \%)$ were housewives, but $90 \quad(23.3 \%)$ were government employees, and $88(22.8 \%)$ were private employees, while 63 $(16.3 \%)$ were laborers, and $20(5.2 \%)$ were merchants. The educational status of a husband who cannot read and write was $60(15.1 \%)$, and $58(15.0 \%)$ could read and write, while $21(5.4 \%)$ were primary, and $46(11.9 \%)$ were secondary level, but $25(6.5 \%)$ were above secondary, although 12 (3.1\%) were Quranic level.

The educational status of the wives varied. Among 59 $(15.3 \%)$ could read and write, but $50(13.0 \%)$ could not read and write. Of those, $19(4.9 \%)$ were primary level, but
$31(8.0 \%)$ were secondary, while $3(0.8 \%)$ were above secondary. The occupational status of the husbands was 43 (11.1\%). The proportion of daily laborers was 57 (14.8\%), mainly working as private employees, but the merchant proportion was $54(14.0 \%)$, and this is the $46(11.9 \%)$ were government employees. Meanwhile, 16 (4.1\%) were farmers, so most of the husbands were private employees. The family size was $135(35.0 \%) 3-4$, while 127 (32.9\%) were 2-3 people, but $86(22.3 \%)$ were $4-10$ people. However, the largest family size was 135 (35.0\%), between 3-4 people per household. Regarding monthly income, 138 (35.8\%) were around 50-100 dollars, 175 (45.3\%) were $100-200$, and $73(18.9 \%)$ were more than 200, while 86 $(22.3 \%)$ were $4-10$.

Table 1. Socio-demographic characteristics.

\begin{tabular}{|c|c|c|c|}
\hline Variables & Frequencies (\%) & Variables $(\%)$ & Frequencies (\%) \\
\hline Age & & Education of husband & \\
\hline $16-39$ years & $258(66.8)$ & Can't read and write & $60(15.1)$ \\
\hline $40-86$ years & $128(33.2)$ & Can read and write & $58(15.0)$ \\
\hline Sex of respondent & & Primary (grade 1) & $21(5.4)$ \\
\hline Male & $166(43.0)$ & Secondary (9-12) & $46(11.9)$ \\
\hline Female & $220(57.0)$ & Education of wife & \\
\hline Marital status & & Can't read and write & $50(13.0)$ \\
\hline Single & $98(25.4)$ & Can read and write & $59(15.3)$ \\
\hline Married & $221(57.3)$ & Primary (grade 1) & $19(4.9)$ \\
\hline Divorced & $36(9.3)$ & Secondary (9-12) & $31(8.0)$ \\
\hline Widowed & $31(8.0)$ & Above secondary & $3(0.8)$ \\
\hline Educational status & & Occupation of husband & \\
\hline Can't read and write & $151(39.1)$ & Farmer & $43(11.1)$ \\
\hline Can read and write & $86(22.3)$ & Government employee & $46(11.9)$ \\
\hline Secondary (9-12) & 4311.1) & Daily laborer & $54(14.0)$ \\
\hline University & $37(9.6)$ & Merchant & $16(4.1)$ \\
\hline Current occupation & & Family size & \\
\hline Housewife & $121(31.3)$ & $2-3$ & $127(32.9)$ \\
\hline Government employee & $90(23.3)$ & $3-4$ & $135(35.0)$ \\
\hline Private employee & $88(22.8)$ & $4-10$ & $86(22.3)$ \\
\hline Laborer & $63(16.3)$ & $10>$ & $38(9.8)$ \\
\hline Merchant & $20(5.2)$ & Monthly income & \\
\hline \multirow[t]{3}{*}{ Farmer } & $4(1.0)$ & $50-100$ & $138(35.8)$ \\
\hline & & $100-200$ & $175(45.3)$ \\
\hline & & More than 200 & $73(18.9)$ \\
\hline
\end{tabular}

Table 2. Water Accessibility and environmental characteristics.

\begin{tabular}{|c|c|c|c|}
\hline Variable & Frequency (\%) & Variable & Frequency (\%) \\
\hline Did you access water? & & Did you met Challenges? & \\
\hline Yes & $386(100.0)$ & No & $214(55.4)$ \\
\hline Distance of water & & Yes & $172(44.6)$ \\
\hline between 30 and $50 \mathrm{~m}$ & $133(34.5)$ & Source water for general use & \\
\hline More than $50 \mathrm{~m}$ & $143(37.0)$ & Water tap & $220(57.0)$ \\
\hline Less than $30 \mathrm{~m}$ & $110(28.5)$ & Well & $140(36.3)$ \\
\hline Piped water & $26(6.7)$ & & \\
\hline Source of drink water & Is this source protected? & & \\
\hline Water tap & $149(38.6)$ & Yes & $299(77.5)$ \\
\hline Well & $76(19.7)$ & No & $87(22.5)$ \\
\hline Piped water & $158(40.9)$ & Does the tap leak? & \\
\hline Surface water & $3(0.8)$ & Yes & $126(32.6)$ \\
\hline Days of water is off & & No & $260(67.4)$ \\
\hline One day per week & $255(66.1)$ & & \\
\hline Two days per week & $113(29.3)$ & & \\
\hline More than two days & $18(4.7)$ & & \\
\hline
\end{tabular}




\subsection{Water Accessibility and Environmental Characteristics}

This study found that $386(100 \%)$ of the respondents could access water. The distance of the water source from household $143(37.0 \%)$ was more than 50 meters. In contrast, 30 and 50 meters were $133(34.5 \%)$ of the water distance while 110 $(28.5 \%)$ were less than 30 meters. The water tap is around 149 (38.6\%). Even though 79 (19.7\%) were obtained from the well, $158(40.9 \%)$ were obtained from piped water, but $3(0.8 \%)$ got surface water. Due to this reason, the most consumed water source is piped water at $158(40.9 \%)$. Approximately one day per week, water is off for 255 (66.1\%), and 113 (29.3\%) two days per week, while $18(4.7 \%)$ are off more than two days. Most of the time, water off is one day per week, 255 (66.1\%). Regarding challenges of water accessibility, 214 (55.4\%) were answered no, while $172(44.6 \%)$ were answered yes. Around $220(57.0 \%)$ got water from the water tap. The $140(36.3 \%)$ get their water from a well, while $26(6.7 \%)$ get it from a pipe, and $299(77.5 \%)$ answered yes, while $87(22.5 \%)$ answered no. We asked the participants if their tap was leaking; $260(67.4 \%)$ answered no, and 126 (32.6\%) answered yes, respectively.

\subsection{Water Storage and Practice at the Household Level}

In Table 3, most containers for water storage at the household level were plastic container 291 (75.4\%). Approximately 48 (12.4\%) were used clay pots, and 47 (12.2\%) were cement tanks. Most containers used for water storage in households are plastic containers because of their availability and low cost in the market. Residents who have containers were 227, (58.8\%). Among the $114(29.5 \%)$ had two containers. Nearly $43(11.1 \%)$ had three containers. Only two $(0.5 \%)$ had more than three containers. In the Warta-Nabada district, residents had one container out of 227 (58.8\%) with the proper capacity. For $144,(37.3 \%)$ were practiced $\leq 25$ liters, and $242(62.7 \%)$ were $>25$ liters. Regarding the condition of containers, $280(72.5 \%)$ were clean, while $106(27.5 \%)$ were dirty. More than $230(59.6 \%)$ covered their containers, and 156 (40.4\%) answered no as well. Children use their cups for drinking water. The $163(42.2 \%)$ and $138(35.8 \%)$ were used with one single cup. The $59(35.8 \%)$ were used with their hands, $26(6.7 \%)$ were used with their mouth.

Table 3. Water storage and practice at household level.

\begin{tabular}{llll}
\hline Variable & Frequency (\%) & Variable & Frequency (\%) \\
\hline Type of container & & Container wash & $105(27.2)$ \\
Plastic container & $291(75.4)$ & No & $281(72.8)$ \\
Clay pot & $48(12.4)$ & Yes & $41(10.6)$ \\
Cement tank & $47(12.2)$ & Cleaning water container & $122(31.6)$ \\
Containers have & & Daily & $91(23.6)$ \\
One & $227(58.8)$ & Several times per week & $19(4.9)$ \\
Two & $114(29.5)$ & One a week & $8(2.1)$ \\
Three & $43(11.1)$ & Once a month & $26(6.7)$ \\
More than three & $2(0.5)$ & Don't know & $83(21.5)$ \\
Capacity of container & & Materials wash container & $136(35.2)$ \\
$\leq 25$ Liters & $144(37.3)$ & Vegetation & $36(9.3)$ \\
$>25$ Liters & $242(62.7)$ & Chemicals & \\
Condition of containers & & Only water & $273(70.7)$ \\
Clean & $280(72.5)$ & Water with soap & $113(29.3)$ \\
Dirty & $106(27.5)$ & Did you fetch the water? & \\
Did you cover container? & & No & $16(4.1)$ \\
No & $230(59.6)$ & Yes & $98(25.4)$ \\
Yes & $156(40.4)$ & Container use for fetching & \\
Children drink & & Clay pot & $207(53.6)$ \\
With their own cups & $163(42.2)$ & Jerri can & $179(46.4)$ \\
With one single cup & $138(35.8)$ & Did you wash your hands? & \\
With their hands & $59(35.8)$ & No & $39(10.1)$ \\
With their mouths & $26(6.7)$ & Yes & $48(12.4)$ \\
Days water is stored & & Times fetch per day & $23(6.0)$ \\
For two days & $124(32.1)$ & One & $4(1.0)$ \\
For three days & $140(36.3)$ & Two & Three \\
More than three days & $122(31.6)$ & More than Four & \\
& & &
\end{tabular}

Therefore, most children use it when they drink their cups, $163(42.2 \%)$. The number of days for water stored in the household was two days, 124 (32.1\%). Also, 140, (36.3\%) were stored for three days in their water. For these results, 122 $(31.6 \%)$ were stored for more than three days compared to the above results. The longest days were three days, 140 (36.3\%). When asked if they washed their containers regularly, 281 $(72.8 \%)$ said yes, and $105(27.2 \%)$ said no. The study participants cleaned their water containers daily to keep them safe. Households of $41(10.6 \%)$ and $122(31.6 \%)$ were cleaned multiple times per week, respectively. Materials used for container washing, $1136(35.2 \%)$ were used only for water for cleaning, while $26(6.7 \%)$ were used for vegetation. Less than $83(21.5 \%)$ were used as chemicals, and $36(9.3 \%)$ were used as soap with water. More than $273(70.7 \%)$ answered no in the water fetching test, while $113(29.3 \%)$ answered yes. The 
respondents who use containers for fetching, 98 (25.4\%) use Jerri cans, and 16 (4.1\%) use clay pots. Twenty-four percent of the Jerri cans are the most usable water fetching in the study site. Furthermore, when it came to washing their hands, 207 (53.6\%) did not wash their hands after dipping them in water, while 179 (46.4\%) did so regularly. For instance, 39 (10.1\%) were fetched one time, with $48(12.4 \%)$ fetched two times, instead of $23(6.0 \%)$ fetched three times, while $4(1.0 \%)$ were fetched more than four times.

\subsection{The Knowledge Level and Methods of Household Water Treatment}

Table 4 shows the majority of the respondents have known the household water treatment and its practices properly 272 $(70.5 \%)$, but $114(29.5 \%)$ was not known how to practice household water treatment. Regarding the objectives of household water treatment, $163(42.2 \%)$ answered prevent diarrheal disease, while $76(19.7 \%)$ answered reduces infant mortality. In addition, these respondents know how to reduce water-borne diseases 32 (8.3\%). Moreover, 262 (67.9\%) of the participants did not have knowledge of the causes of water contamination. The $124(32.1 \%)$ of the participants know the underlying causes of water contamination, and 226 (58.5\%) answered diarrheal disease, while 105 (27.2\%) answered infant mortality, $36(9.3 \%)$ answered malnutrition, and $19(4.9 \%)$ answered water borne-diseases. Of these, 219 (56.7\%) used household treatment methods, and 80 (20.7\%) were not used. Methods $91 \quad(23.6 \%)$ were used for chlorination, 61 (15.8\%) for boiling, 45 (11.7\%) for filtration, and $22(5.7 \%)$ for solar disinfection. In addition, the majority of the participants were practiced water for chlorination 91 $(23.6 \%)$ as treatment.

Table 4. Knowledge level and methods of household water treatment.

\begin{tabular}{|c|c|c|c|}
\hline Variable & Frequency (\%) & Variable & Frequency (\%) \\
\hline Knowledge of household & & Contamination results & \\
\hline No & $114(29.5)$ & Diarrheal disease & $226(58.5)$ \\
\hline Yes & $272(70.5)$ & Infant mortality & $105(27.2)$ \\
\hline Objectives of HWT & & Malnutrition & $36(9.3)$ \\
\hline Prevent diarrheal disease & $163(42.2)$ & Water-borne diseases & $19(4.9)$ \\
\hline Reduces infant mortality & $76(19.7)$ & Did you use any method & \\
\hline Reduces water porn disease & $32(8.3)$ & No & $80(20.7)$ \\
\hline No & $124(32.1)$ & How many methods use? & \\
\hline \multirow[t]{4}{*}{ Yes } & $262(67.9)$ & Chlorination & $91(23.6)$ \\
\hline & & Boiling & $61(15.8)$ \\
\hline & & Filtration & $45(11.7)$ \\
\hline & & Solar Disinfection & $22(5.7)$ \\
\hline
\end{tabular}

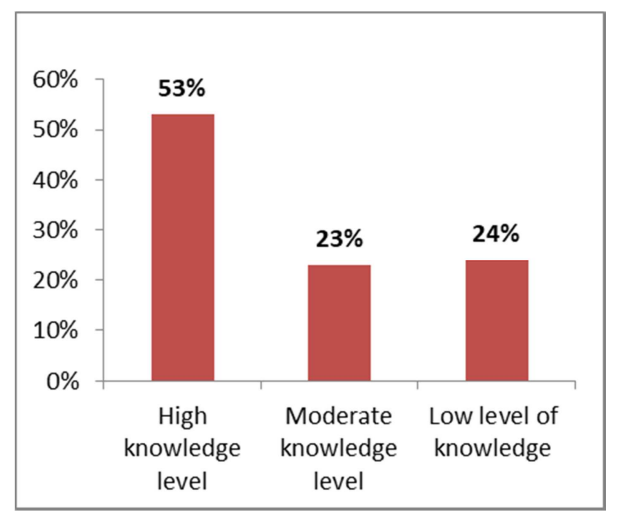

Figure 1. Level of knowledge.

Figure 1. Knowledge scores of household-level water treatment were divided into three main categories: high knowledge level, moderate knowledge level, and low-level knowledge. The high knowledge level was 205 (53\%), the moderate knowledge level was 88 (23\%), and 93 (24\%) had a low level of knowledge, respectively. However, the majority of the residents in the study location have sufficient knowledge of household water treatment. Figure 2. Focused on the practice level of household water treatment, two categories based on which are good practice and poor practice. Also, the group of good practices was 281 (73\%), while the poor practices group was $105(27 \%)$. Therefore, the majority of residents practiced the accurately.

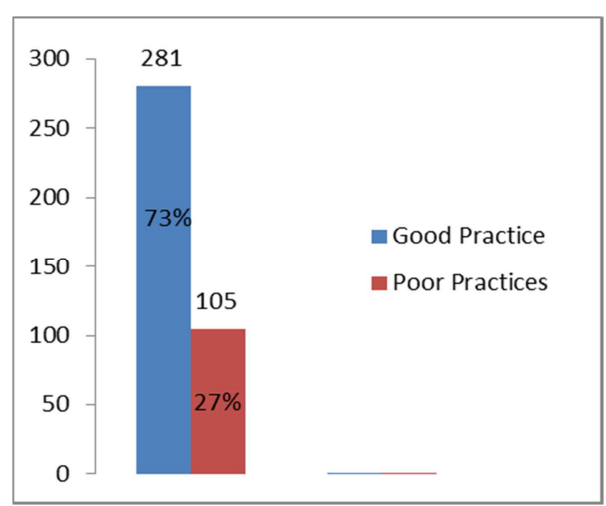

Figure 2. Knowledge practices.

\subsection{Association Between Socio-demographic and Knowledge Level}

The results in table 5 show that the husband's age, marital status, occupation, and education were found to be significant. Participants aged 16 to 39 years have more knowledge than 40 to 86 years (OR $0.652,95 \%$ CI $0.424-0.999$ ).

Unmarried respondents have more knowledge than married respondents (OR 0.342, 95\%CI .212- .554). The 
employed respondents have more knowledge than the unemployed respondents (OR 1.611, 95\%CI 1.040-2.495).
The educated husbands have more knowledge than the uneducated husbands (OR 0.534, 95\%CI .305- .936).

Table 5. Association between socio-demographic and knowledge level.

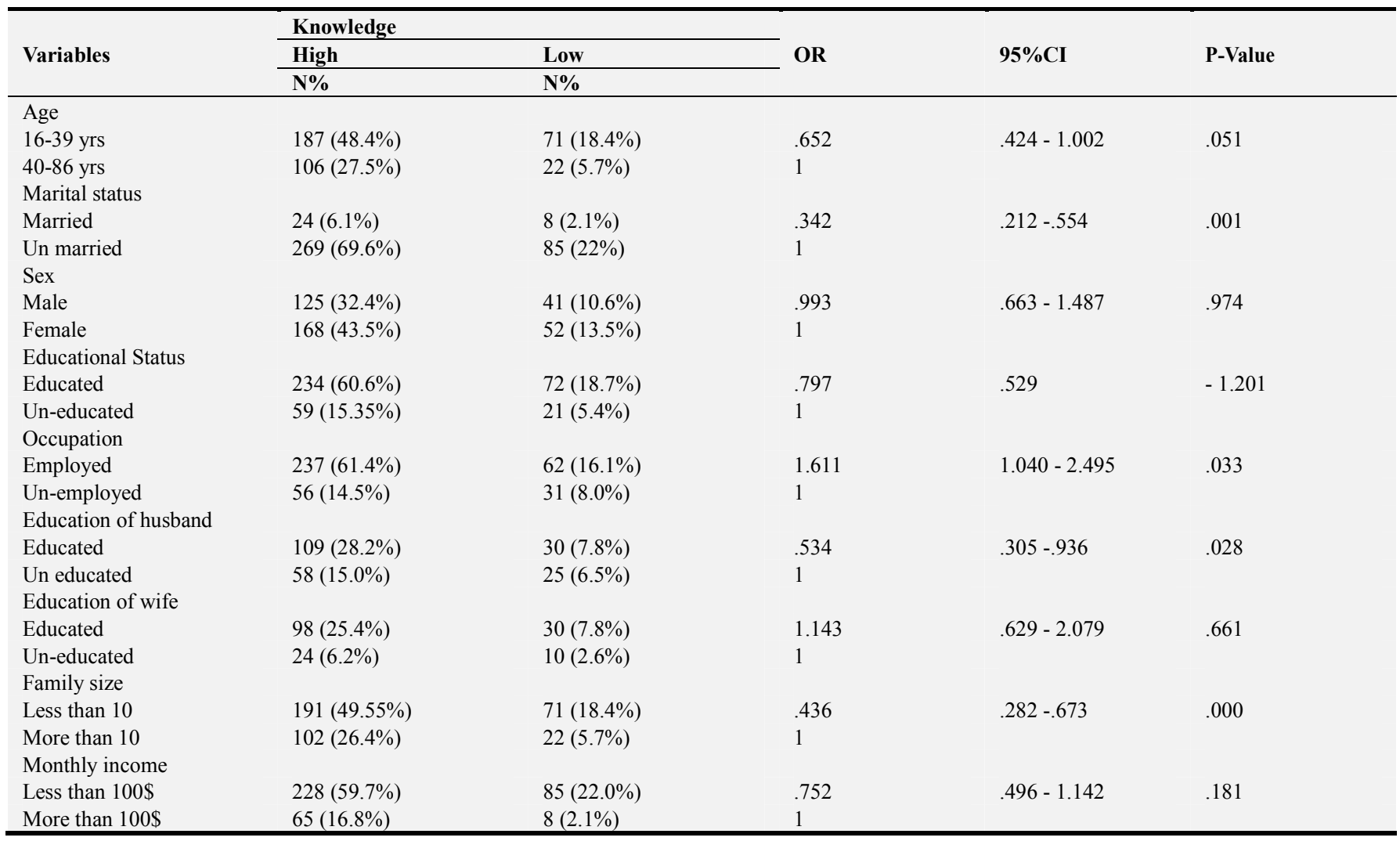

*= Significant where $\mathrm{P}$-value $<0.05, \mathrm{OR}=$ Odds ratio, $\mathrm{CI}=$ Confidence interval.

\section{Discussions}

The majority of respondents, 291 (75.4\%), used plastic containers for water storage at a household level, while 48 $(12.4 \%)$ used clay pots, followed by $47(12.2 \%)$ cement tanks. Most containers used for water storage are plastic containers because of their availability and low cost. Residents who have containers were 227 (58.8\%). Among 114 (29.5\%), two containers were found. The number of people who practice boiling water was relatively low $(15.8 \%)$. Compared to another study conducted in India that highlighted around $60 \%$ of the knowledge of boiling water, followed by chlorination $(27 \%)$ and membrane filtering at $(22.4 \%)$ [13]. In the WartaNabada district, the filtration rate was $(11.7 \%)$ and only (5.7\%) used solar disinfection for drinking and domestic water. Another study revealed that the level of knowledge on water chlorination was also comparable $(23.6 \%)$ with that of the Myanmar study (10\%) [14]. However, other research conducted in Somalia revealed that there was no variable association between latrine availability and household water treatment practices [11].

On the other hand, the respondents' ages in this study were between 16 and 39 years old, which has more knowledge than those ages between 40 and 86 years old (OR 0.652, 95\%
CI 0.424-1.002). The unmarried participants have better knowledge than the married respondents (OR 0.342, 95\%CI. 212-554). The employed participants have good knowledge compared to the unemployed respondents (OR 1.611, 95\% CI 1.040-2.495). Therefore, educated husbands have more knowledge than uneducated husbands in household water treatment practices (OR 0.534, 95\%CI .305- .936).

However, nearly half of mothers and caregivers (49.3\%) understood household water treatment methods [15]. The current study highlighted the use of the chlorination method was $(23.6 \%)$. The other study highlights that chlorinated water was associated with a $48 \%$ reduction in diarrhea [16]. These results indicate that the chlorination of water can be successfully carried out locally to improve the health of the population. The majority of the participants are interested in household water treatment $(62.8 \%)$. The findings also revealed that $(43.0 \%)$ of respondents were male, while $(57.0 \%)$ were female. The population sample that collected the data was categorized into single, married, divorced, and widowed. The (39.1\%) could not read, while $86(22.3 \%)$ could read and write, but $69(17.9 \%)$ were at the primary level, and $43(11.1 \%)$ were secondary. Besides, the lowest portion, $37(9.6 \%)$, were universities. The majority of respondents $(31.3 \%)$ were housewives, while 90 (23.3\%) were government employees, and $(22.8 \%)$ were private- 
sector employees. Furthermore, 16.3\% were laborers, 5.2 percent were merchants, and farmers accounted for the minor proportion, around $4.0 \%$. The husband's educational status (15.1\%) was that he could not read and write, while (15.0\%) could not read and write. The level of primary schools was $(5.4 \%)$ and $(11.9 \%)$ were secondary level. Nevertheless, $3.1 \%$ were Quranic level.

\section{Conclusion and Recommendation}

According to these results, it can be concluded there were significant variables for age, marital status, occupational, and education of the husband of the household water treatment practices in Mogadishu. When there was a high risk of acute water diarrhea or cholera, household water treatment methods were primarily related to drinking water chlorination, boiling, and filtration. The group of good practices was $(73 \%)$, while the poor practices group was (27\%). Nevertheless, the majority of residents practice the proper way. This study's findings suggest both a need for improving the handling and storage practices of drinking water at a household level and methods of water treatment practices for reducing health risks related to the drinkingwater supply.

\section{References}

[1] UNICEF and WHO: World Health Organization: Progress on drinking water and sanitation: update, Nueva York: UNICEF, available at: http://apps.who.int/iris/bitstream/10665/81245/1/ 9789241505390_eng.pdf (last access: 5 April 2016), 2012.

[2] Edema OjomoaMark Elliottab Lorelei Good year c Michael Forsond Jamie Bartrama. 2015. Sustainability and scale-up of household water treatment and safe storage practices: Enablers and barriers to effective implementation. Volume 218, Issue 8, November 2015, Pages 704-713.

[3] Brown Sobsey, M. D. and Loomis, D.: Local drinking water filters reduce diarrheal disease in Cambodia: a randomized, controlled trial of the ceramic water purifier, Am. J. Trop. Med. Hygiene 79, 394-400, 2008.

[4] Sobsey et al., 2008). Sobsey, M. D., Stauber, C. E., Casanova, L. M., Brown, J. M., and Elliott, M. A.: Point of use household drinking water filtration: a practical, effective solution for providing sustained access to safe drinking water in the developing world, Environ. Sci. Technol., 42, 42614267,2008
[5] Subrat K. Pradhan, Upasana Sinha, Durga M. Satapathy, Amit P. Swain, Rudra P. Mishra. 2018. Assessment of household water treatment and storage practices. DOI: http://dx.doi.org/10.18203/2394-6040.ijcmph2018076.

[6] Duke, W., Nordin, R., Baker, D., and Mazumder, A. The use and performance of BioSand filters in the Artibonite Valley of Haiti: a field study of 107 households, Rural Remote Health, 6, $570,2006$.

[7] Heins broek, A. and Peters, T. Solar Water Disinfection in Developing Countries, CIE5421 Water \& Health Technology Review Essay, 2014.

[8] Meierhofer, R. and Landolt, G.: Factors supporting the sustained use of solar water disinfection - Experiences from a global promotion and dissemination programme, Desalination, 248, 144-151, 2009.

[9] Nath KJ, Bloomfield SMJ. Household water storage, handling and point-of-use treatment. Vol. 34, International Scientific Forum on Home Hygiene. 2016.

[10] Clasen T, Cairncross S. Editorial: household water management: refining the dominant paradigm. Trop Med Int Health. 2004; 9 (2): 187-191. doi: 10.1046/j.13653156.2003.01191.

[11] Abdirizak Mohamud Yusuf. Ali M. Omer. Sayid-omer Yasin. Hussein Jama Had. Mohamed Osman Ali. Prevalence And Factors Associated With Diarrhea Among Children Aged 0-59 Months In Badbaado Camp In Mogadishu, Somalia. Researchjournali's Journal of Public Health Vol. 4 | No. 11 November $\mid 2018$.

[12] A. G. Misati 2016: Published online 2016 Jun 21. doi: 10.1007/s12199-016-0547-x PMCID: PMC5112191 PMID: 27329278 Household safe water management in Kisii County, Kenya.

[13] Bikes D. Bitew, 2017. Knowledge, Attitude, and Practice of Mothers/Caregivers on Household Water Treatment Methods in Northwest Ethiopia: A Community-Based Cross-Sectional Study.

[14] Mahfouz AA, 1995. Impact of chlorination of water in domestic storage tanks on childhood diarrhoea: a community trial in the rural areas of Saudi Arabia.

[15] Subrat K. Pradhan, (2017). Assessment of household water treatment and storage practices.

[16] UNICEF, 2011. Knowledge, Attitude and Practice Study into Water, Sanitation and Hygiene in 24 Townships of Myanmar Available at: http://www.burmalibrary.org/docs17/WASHMyanmar_\%202011_KAP_Study-red. Accessed January 30, 2017. 